\title{
ANÁLISIS COMPARATIVO ENTRE CLOROFILA-A SATELITAL E IN SITU EN EL ECOSISTEMA DE AFLORAMIENTO PERUANO, ENTRE 1998 - 2007
}

\section{COMPARATIVE ANALYSIS BETWEEN SATELLITE AND IN SITU CHLOROPHYLL-A IN THE PERUVIAN UPWELLING ECOSYSTEM, BETWEEN $1998-2007$}

\author{
Daniel Quispe ${ }^{1}$, Ruth Calienes ${ }^{1}$, Jorge Tam ${ }^{1}$, Michelle Graco ${ }^{2}$, Jesús Ledesma ${ }^{2}$, Georgina Flores ${ }^{2}$, David Correa ${ }^{1}$ \\ y Carlos Quispe ${ }^{1}$
}

\begin{abstract}
Resumen
El ecosistema de afloramiento peruano posee una alta variabilidad espacial y temporal en su producción primaria, que puede ser inferida a partir de observaciones de clorofila-a in situ y vía satélite. Las diferencias entre la clorofila-a in situ del IMARPE $\left(\mathrm{Cla}_{\mathrm{i}}\right)$ y clorofila-a satelital del SeaWiFS $\left(\mathrm{Cla}_{\mathrm{s}}\right)$ fueron estudiadas usando valores coincidentes en el espacio y tiempo, para conocer la relación entre $\mathrm{Cla}_{\mathrm{i}} \mathrm{y} \mathrm{Cla}_{\mathrm{s}}$ a partir de un modelo de regresión lineal simple. Asimismo, se determinó el ajuste entre ambas observaciones en las diferentes estaciones del año, calculando la raíz del cuadrado medio del error. La hipótesis fue que las observaciones de $\mathrm{Cla}_{\mathrm{i}}$ sobreestiman la variabilidad de $\mathrm{Cla}_{\mathrm{i}}$ y que en verano hay un mejor ajuste entre los valores de $\mathrm{Cla}_{\mathrm{i}} \mathrm{y} \mathrm{Cla}_{\mathrm{s}}$, respecto a las otras estaciones del año. La ecuación general que relaciona ambas variables fue: $\mathrm{Cla}_{\mathrm{i}}=0.9925$ $\mathrm{Cla}_{\mathrm{s}}{ }^{0.9055}$. Se encontró que el ajuste con transformación $\log _{10}$ fue máximo en primavera $(\mathrm{r}=0.84$, $\mathrm{RCME}=0.31)$ y mínimo en otoño $(\mathrm{r}=0.72$, RCME $=0.37)$. La clorofila-a fue sobreestimada en condiciones de verano y otoño, y subestimada en invierno y primavera por el SeaWiFS.

Palabras clave: clorofila-a, SeaWiFS, modelo de regresión, afloramiento, Perú.
\end{abstract}

\begin{abstract}
The Peruvian upwelling ecosystem has a high spatial and temporal variability in its primary production that could be inferred from in situ and satellite observations of chlorophyll. The differences between in situ $\left(\mathrm{Cla}_{\mathrm{i}}\right)$ from IMARPE and satellite chlorophyll-a $\left(\mathrm{Cla}_{\mathrm{s}}\right)$ from SeaWiFS data were studied using spatial and temporal coincident values, to know the relationship between $\mathrm{Cla}_{\mathrm{i}}$ and $\mathrm{Cla}_{\mathrm{s}}$ values from a simple linear regression model. Also, the goodness of fit between both observations during each season was calculated using the square root mean error. The hypothesis was that the $\mathrm{Cla}_{\mathrm{s}}$ observations overestimated the $\mathrm{Cla}_{\mathrm{i}}$ values and that in summer there is a better goodness of fit between $\mathrm{Cla}_{\mathrm{i}}$ and $\mathrm{Cla}_{\mathrm{s}}$ data, compared to other seasons. The general equation obtained relating both variables was: $\mathrm{Cla}_{\mathrm{i}}=0.9925 \mathrm{Cla}_{\mathrm{s}}{ }^{0.9055}$. The goodness of fit between both data sources with a $\log _{10}$ transformation was higher in spring $(r=0.84$, RCME $=0.31$ ) than in summer $(r=0.82$, RCME $=0.33$ ). The chlorophyll-a was overestimated in summer and fall, while it was underestimated in winter and spring by SeaWiFS satellite.
\end{abstract}

Key words: chlorophyll-a, SeaWiFS, regression model, upwelling, Perú.

\section{Introducción.}

El sistema de afloramiento marino frente a Perú, está asociado a una importante actividad socioeconómica sustentada por altos niveles de productividad primaria, los cuales constituyen la base de la alimentación de los subsiguientes eslabones en la cadena trófica. El entendimiento de la estructura y funcionamiento del sistema aún es un reto que se va superando con la aplicación de técnicas que integran con mayor aproximación el efecto de diferentes factores del ecosistema marino sobre indicadores de productividad, como los observados vía satélite por el Sea-viewing Wide Field-of-view Sensor (SeaWiFS).
El ecosistema marino frente a Perú es evaluado periódicamente a través de prospecciones oceanográficas y la determinación de clorofila-a in situ es una de las variables que se toma en cuenta para describir las condiciones de productividad en el sistema. A pesar de su irregular frecuencia se ha llegado a conocer algunas características asociadas a su distribución espacial y temporal, estableciendo patrones promedio de distribución de clorofila-a en superficie, relacionados con la distribución de nutrientes y producción primaria de fitoplancton, así como la descripción del ciclo estacional en términos de biomasa de fitoplancton, identificándose como 
rango a concentraciones mayores a $2 \mathrm{mg} \cdot \mathrm{m}^{-3}$, dentro de las 50 mn (Calienes et al., 1985), y su variabilidad en condiciones El Niño, por ejemplo durante el otoño de 1998 (Flores et al., 1998), como también la presencia de importantes núcleos de productividad, asociados a las principales zonas de afloramiento como son Callao, Chimbote y Chicama (Flores et al., 2001). Todos estos aspectos se presentan en múltiples reportes internos de prospecciones oceanográficas conducidas por el Instituto del Mar del Perú (IMARPE).

Recientemente el típico patrón de distribución espacial de clorofila-a fue comparado en base a datos de satélite e in situ, hallándose que las máximas concentraciones de clorofila-a (5-10 mg.m ${ }^{-3}$ ) decrecen gradualmente hacia fuera de la costa con valores aproximadamente de 0.5 a 1.5 mg. $\mathrm{m}^{-3}$ entre los 200$300 \mathrm{~km}$ de la costa, presentando los datos satelitales una zona de alta productividad más estrecha que con las observaciones de data in situ (Echevin et.al., 2008). Se sabe que hay mayor clorofila-a frente a la costa peruana en verano e inicios de otoño, y menor en invierno, cuando existe una correlación negativa con los nutrientes (Calienes et al., 1985; Thomas et al., 2001; Pennington et al., 2006; Echevin et al., 2008).

En otras latitudes, como al norte de Chile $\left(18^{\circ} 21^{\prime} \mathrm{S}\right.$ - $24^{\circ} \mathrm{S}$ ), en base a imágenes de clorofila-a se reportó un gradiente espacial de este a oeste, cuyas altas concentraciones en zonas costeras estuvieron asociadas con eventos de afloramiento de aguas frías (Pizarro et al., 1994).

Chávez (1995) comparó la relación entre la clorofila-a a partir de observaciones in situ y vía satélite del Coastal Zone Color Scanner (CZCS), hallando que la concordancia entre ambas fuentes para el sistema de afloramiento peruano era bastante pobre presentando en promedio que la clorofila-a in situ fue 3.8 veces más grande que la estimada por el satélite, a diferencia del cociente en California que fue de 1.1. Las concentraciones en Perú fueron mayores que en California. Sin embargo en estudios posteriores se ve que estas discordancias fueron cambiando, reportándose en algunos estudios que los valores satelitales de clorofila-a están sobreestimados. Por otra parte el método frecuentemente usado para determinaciones de clorofila-a in situ (i.e. fluorométrico con acidificación), también podría dar resultados sobrestimados o subestimados (Sin-Jae, 2002), debido a diferentes factores, como la variabilidad de las condiciones atmosféricas (Chin et al., 2009), a gradientes en la composición de especies de fitoplancton (Chávez, 1995) y a factores asociados a la variabilidad estacional de las Propiedades Opticas Inherentes (IOP), (Loisel et al, 2010).

Cuando se intenta deducir las características de variabilidad espacio-temporal de la producción primaria en el sistema de afloramiento peruano, a partir del pigmento fotosintético predominante de organismos autótrofos, desde observaciones in situ y vía satélite (SeaWiFS), el problema es que aún hay diferencias entre el resultado de ambas observaciones en términos de mg.m ${ }^{-3}$ de clorofila-a, sin embargo constantemente se están realizando intercalibraciones entre observaciones in situ y derivadas de satélite (O’Reilly, 2000; Maritorena, 2002; Werdell et al., 2007; Pan et al., 2010), por lo que se espera desarrollar algoritmos específicos a las condiciones locales de cada región, en especial del mar peruano.

La información obtenida a partir de éste estudio, permitirá para éste sistema, comparar los resultados procedentes de ambas fuentes, inferir la variabilidad de clorofila-a con mayor resolución espacio-temporal y validar los modelos asociados a la producción primaria.

En vista de esta problemática, y considerando la menor nubosidad en verano, en el presente trabajo se plantearon las siguientes hipótesis: (i) la $\mathrm{Cla}_{\mathrm{s}}$ sobreestiman a la $\mathrm{Cla}_{\mathrm{i}} \mathrm{y}$ (ii) en condiciones de verano hay un mejor ajuste entre los valores de $\mathrm{Cla}_{\mathrm{i}} \mathrm{y} \mathrm{Cla}$, que en las demás estaciones del año, debido a la variabilidad estacional de las Propiedades Opticas Inherentes.

El objetivo general del trabajo fue comparar concentraciones diarias de clorofila satelital e in situ en el sistema de afloramiento peruano, entre 1998 y 2007. Los objetivos específicos fueron: (i) seleccionar los valores de $\mathrm{Cla}_{\mathrm{i}}$ y $\mathrm{Cla}_{\mathrm{s}}$ que coinciden espacial y temporalmente; (ii) determinar una ecuación de regresión lineal simple que permita conocer la relación entre $\mathrm{Cla}_{\mathrm{i}}$ y Cla $\mathrm{Cl}_{\mathrm{s}}$; (iii) comparar y determinar el ajuste entre las observaciones de $\mathrm{Cla}_{\mathrm{i}}$ y $\mathrm{Cla}_{\mathrm{s}}$ en los diferentes períodos estacionales del año.

\section{Materiales y métodos. \\ Datos}

En el sistema de afloramiento peruano, se evaluaron reportes de clorofila in situ, del nivel superficial del mar y estimados vía satélite (SeaWiFS), para el periodo entre 1998 y 2007. La Cla fue determinada en base a muestras colectadas a lo largo de la costa de Perú, usando aproximadamente en el 90 \% de los casos la técnica fluorométrica de HolmHansen et al. (1965), que corrige el efecto de feopigmentos, y en el $10 \%$ de los casos el método espectrofotométrico de acuerdo a Strickland \& Parsons (1968), por la Unidad de Investigaciones en Oceanografía Química del Instituto del Mar del Perú (IMARPE). Se obtuvieron datos diarios de $\mathrm{Cla}_{\mathrm{s}}$ del Standard Mapped Image (SMI), producto de nivel 3 (L3), en formato HDF a resolución en coordenadas geográficas de $1 / 12^{\circ}$, y habilitados con MATLAB, y descargados en febrero del 2010, de la base del SeaWiFS Project (http://oceancolor.gsfc.nasa.gov).

\section{Análisis de datos}

La comparación de resultados observados por ambas fuentes, se desarrolló con información 
coincidente en el espacio y tiempo. La coincidencia espacial estuvo referida a la ubicación de datos in situ que se aproximan a la ubicación de data satelital en un radio menor a 2.5 millas náuticas $(\mathrm{mn})$, la calidad de los datos coincidentes fue evaluado con parámetros estadísticos de centralidad y dispersión, verificando características espaciales a través de mapas con escalas de color y traslape en la ubicación (Figura 1), para los datos in situ, valores reportados como cero no fueron considerados en el estudio. La coincidencia temporal estuvo referida a observaciones de clorofilaa en el mismo día.
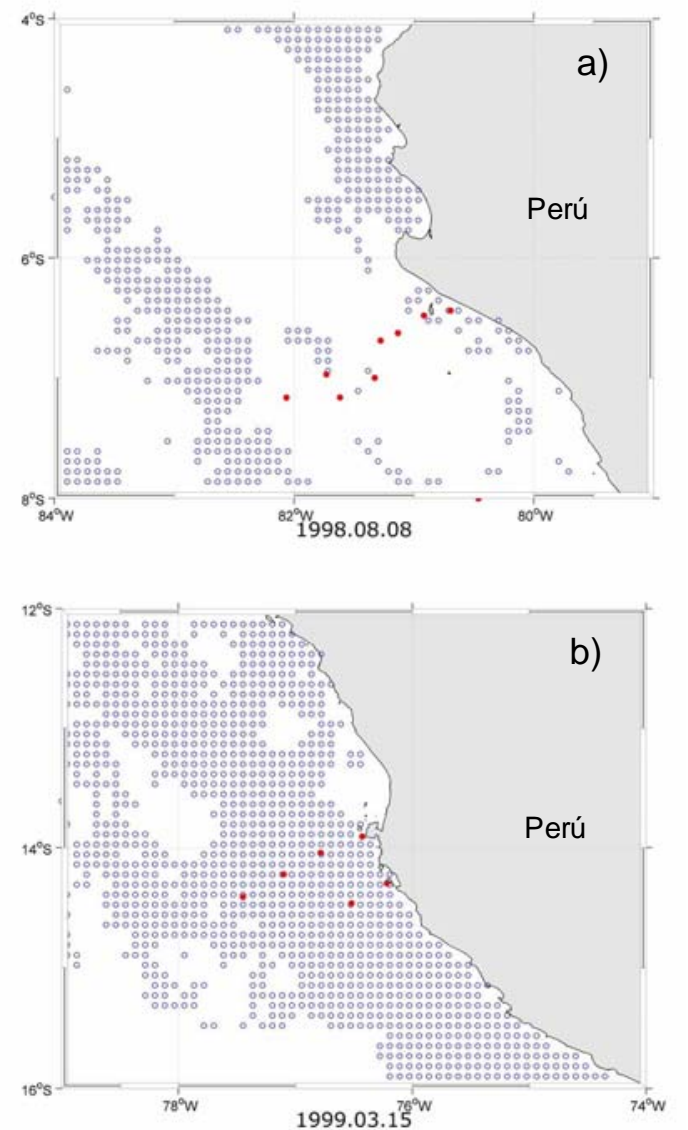

Figura 1. Distribución espacial de algunos datos satelitales (azul) e in situ (rojo) mostrando diferentes características de traslape, a) favorable (verano 1997), b) intermedia (Invierno 1998).

Se realizó una prueba t, con datos transformados con logaritmos en base $10\left(\log _{10}\right)$, para detectar diferencias significativas entre $\mathrm{Cla}_{\mathrm{i}} \mathrm{y} \mathrm{Cla}_{\mathrm{s}}$, así como evaluaciones en base al percentil (p).

La variabilidad estacional fue inferida considerando como verano al primer trimestre del año y así sucesivamente hasta el cuarto trimestre asociado a primavera, para describir características en base a parámetros estadísticos de centralidad, dispersión y correlación, al igual que para la variabilidad interanual.
Se ajustó un modelo de regresión lineal simple con $\mathrm{Cla}_{\mathrm{i}}$ como variable dependiente y $\mathrm{Cla}_{\mathrm{s}}$ como variable independiente. Ambas variables fueron transformadas con $\left(\log _{10}\right)$, con el fin de mejorar la distribución de los errores y satisfacer los supuestos del análisis de regresión.

Para comparar el ajuste entre las observaciones de $\mathrm{Cla}_{\mathrm{i}}$ y $\mathrm{Cla}_{\mathrm{s}}$ en los diferentes periodos estacionales del año, se calculó el cociente de medias en valores con y sin transformación $\log _{10}$, la raíz del cuadrado medio del error (RCME) y el error medio, en términos de porcentaje (EM) (Marrari et al., 2006), los cuales estuvieron definidos de la siguiente manera:

$$
\begin{aligned}
& \text { RCME }=\sqrt{\frac{1}{n} \sum_{i=1}^{n}\left(e_{i}\right)^{2} \times 100} \\
& E M=\bar{e}=\left(\frac{1}{n} \sum_{i=1}^{n} e_{i}\right) \times 100 \\
& e=\frac{\text { Cla }_{s}-\text { Cla }_{i}}{\text { Cla }_{i}}
\end{aligned}
$$

Donde $n$ es el número de pares coincidentes.

Para un conjunto de datos con distribución normal, la RCME podría ser igual a la desviación estándar. Adicionalmente la estimación de los errores fue hecha con los datos transformados con $\log _{10}$.

$$
R C M E_{\log }=\sqrt{\frac{\sum\left[\log \left(C l a_{s}\right)-\log \left(C l a_{i}\right)\right]^{2}}{n}}
$$

$$
E M_{\log }=\frac{\sum\left[\log \left(C l a_{s}\right)-\log \left(C l a_{i}\right)\right]}{n}
$$

\section{Resultados y discusión.}

\section{Distribución espacial de datos coincidentes}

La distribución espacial de datos satelitales fue irregular, conteniendo mayor información en condiciones de verano y menor en invierno. Algunos períodos presentaron una distribución espacial favorable para la evaluación de la variabilidad en el sistema, otros períodos fueron adversos por una 
distribución espacial con poca información satelital atribuida a interferencia por presencia de nubosidad (Figura 1). Sin embargo de un total aproximado a 3800 observaciones in situ, se identificaron 527 observaciones que se presentaron como coincidentes en periodos de un día y en un radio menor de $2.5 \mathrm{mn}$.

La clasificación interanual estuvo constituida en su mayoría entre 10 y 12 \% de datos por año, a excepción de los años 2000, 2001 que tuvieron 6 y $2 \%$ respectivamente y el año 1999 que tuvo más del $17 \%$ de pares de datos coincidentes.

$\mathrm{Al}$ ser clasificados estacionalmente el verano estuvo representado por un $41 \%$ del total de pares coincidentes en contraste al invierno que tuvo solo 8 $\%$ de datos coincidentes, mientras que en otoño y primavera correspondieron 23 y 28 \% respectivamente, de los cuales se pudo observar que en invierno los pares estuvieron ubicados más cerca a la costa que las demás estaciones (Figura 2), siendo distribuidos frente a las costas de Perú, entre aproximadamente 170 - $200 \mathrm{mn}$ mar adentro.

a)
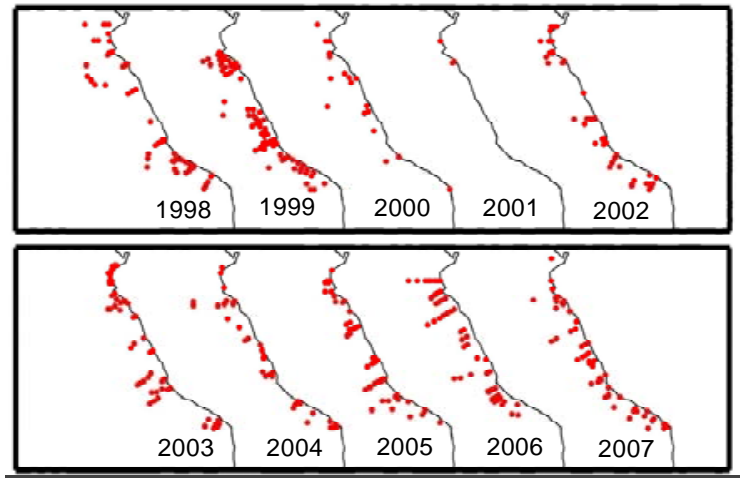

b)

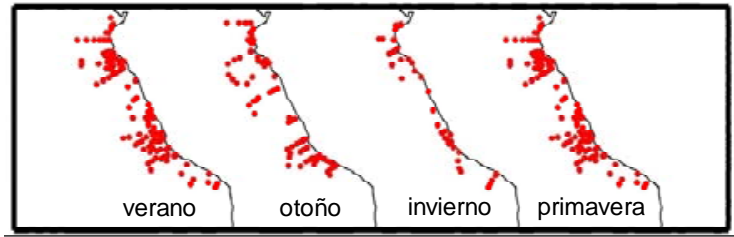

Figura 2. Distribución espacial de pares de datos coincidentes de clorofila-a en relación a la línea de costa de Perú, clasificados en: a) años 1998-2007; b) Estaciones de verano, otoño, invierno y primavera.

\section{Análisis comparativo entre $\mathbf{C l a}_{\mathrm{i}}$ y $\mathbf{C l a}_{\mathrm{s}}$}

Los datos de $\mathrm{Cla}_{\mathrm{i}}$, oscilaron entre 0.03 y 68.90 mg.m ${ }^{-3}$, y los de $\mathrm{Cla}_{\mathrm{s}}$ entre 0.18 y $32.06 \mathrm{mg} \cdot \mathrm{m}^{-3}$. El coeficiente de correlación (r) entre $\mathrm{Cla}_{\mathrm{i}}$ y $\mathrm{Cla}_{\mathrm{s}}$ sin transformación logarítmica de los datos fue de 0.49 (p $<0.01$ ), mientras que el coeficiente de correlación con transformación fue de 0.79 ( $\mathrm{p}<0.01)$.

Una prueba t de diferencia de medias mostró que los valores con transformación $\log _{10}$ de $\mathrm{Cla}_{\mathrm{i}}$ no difieren significativamente de los valores de $\mathrm{Cla}_{\mathrm{s}}(\mathrm{p}=$ $0.50)$.
La relación entre los datos de $\mathrm{Cla}_{\mathrm{i}}$ y $\mathrm{Cla}_{\mathrm{s}}$ se muestra en la Figura 3. La ecuación de regresión lineal simple que permite conocer valores de $\mathrm{Cla}_{\mathrm{i}}$ en función de $\mathrm{Cla}_{\mathrm{s}}$ es la siguiente:

$$
\log _{10} \mathrm{Cla}_{\mathrm{i}}=-0.0033+0.9055 \log _{10} \mathrm{Cla}_{\mathrm{s}}
$$

ó expresado de otra manera como:

$$
\mathrm{Cla}_{\mathrm{i}}=10^{-0.0033} \mathrm{Cla}_{\mathrm{s}}{ }^{0.9055}
$$

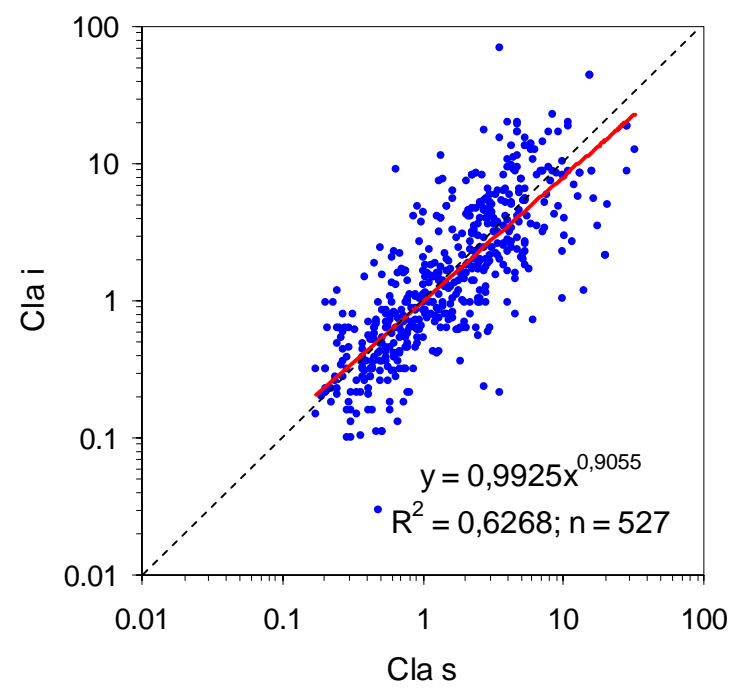

Figura 3. Regresión lineal simple del total de pares coincidentes de clorofila-a satelital $\left(\mathrm{Cla}_{\mathrm{s}}\right)$ frente a clorofila-a in situ $\left(\mathrm{Cla}_{\mathrm{i}}\right)$, transformados con $\log _{10}$.

El cociente $\mathrm{Cla}_{\mathrm{i}}: \mathrm{Cla}_{\mathrm{s}}$ de medias con transformación fue menor a 1 , lo que indica que el satélite sobrestima la clorofila in situ. El mismo cociente con datos no transformados fue mayor a 1 , sin embargo al eliminar los valores extremos del percentil considerando solo los que están entre el rango de p15 y p85, se obtuvo un cociente menor a 1 (Tabla 1).

Comparación estacional de la bondad de ajuste entre $\mathrm{Cla}_{\mathrm{i}} \boldsymbol{y} \mathrm{Cla}_{\mathrm{s}}$

Los resultados de la bondad de ajuste entre $\mathrm{Cla}_{\mathrm{s}} \mathrm{y}$ $\mathrm{Cla}_{\mathrm{i}}$ por periodos estacionales se presentan en la (Tabla 2). El mayor coeficiente de correlación con datos transformados se obtuvo en primavera $(r=0.84$, $\mathrm{p}<0.01$ ), y el menor en condiciones de otoño ( $\mathrm{r}=$ 0.72 , $\mathrm{p}<0.01$ ), mientras que en verano e invierno presentaron un $\mathrm{r}=0.82$ y 0.76 respectivamente, asociados a un $\mathrm{p}<0.01$.

En datos no transformados para verano, otoño, invierno y primavera, los RCME fueron de: 205.20, 182.45, 113.96 y $90.12 \%$ y los EM de: 60.05, 60.49, 30.36, $11.79 \%$ respectivamente.

Los valores de RCME con transformación $\left(\log _{10}\right)$ confirmaron un mejor ajuste entre los datos de $\mathrm{Cla}_{\mathrm{s}} \mathrm{y}$ $\mathrm{Cla}_{\mathrm{i}}$ en primavera $\left(\mathrm{RCME}_{\log }=0.31\right)$ y lo contrario ocurrió en condiciones de invierno $\left(\mathrm{RCME}_{\log }=0.37\right)$, 
mientras que en verano se presentó un ajuste intermedio $\left(\mathrm{RCME}_{\log }=0.33\right)$.

Los errores medios mostraron que durante verano $\left(\mathrm{EM}_{\log }=0.07\right)$ y otoño $\left(\mathrm{EM}_{\log }=0.04\right)$ la clorofila fue sobreestimada desde el satélite, mientras que durante invierno $\left(\mathrm{EM}_{\mathrm{log}}=-0.02\right)$ y primavera $\left(\mathrm{EM}_{\mathrm{log}}=-0.06\right)$ fue subestimada vía satélite, siendo congruente con el cociente menor a 1 obtenido de valores con $\log _{10}$ de $\mathrm{Cla}_{\mathrm{i}}$ : $\mathrm{Cla}_{\mathrm{s}}$, a pesar que a partir del total de datos sin transformación se observó que en verano no concordaban porque tenía un cociente mayor a 1 , al ser evaluados los valores en un rango más estrecho definido por el percentil p1 a p99, el cociente fue menor a 1, llegando a tener concordancia con los valores que determinan que la $\mathrm{Cla}_{\mathrm{s}}$ sobreestima a la $\mathrm{Cla}_{\mathrm{i}}$ 1).

(Tabla

Tabla 1. Cociente $\mathrm{Cla}_{\mathrm{i}}$ : $\mathrm{Cla}_{\mathrm{s}}$, de promedio general y estacional.

\begin{tabular}{llccc}
\hline \multicolumn{2}{l}{ Observaciones } & $\begin{array}{c}\text { media } \\
\text { Clai }\end{array}$ & $\begin{array}{c}\text { media } \\
\text { Clas }\end{array}$ & $\begin{array}{c}\text { cociente } \\
\text { Clai : Clas }\end{array}$ \\
\hline Cla & general & 3.21 & 2.83 & 1.14 \\
& verano & 3.59 & 3.46 & 1.04 \\
& otoño & 2.31 & 2.70 & 0.86 \\
& invierno & 2.59 & 1.82 & 1.43 \\
& primavera & 3.60 & 2.30 & 1.57 \\
\hline \multirow{2}{*}{ og10(Cla) $)$} & general & 0.17 & 0.19 & 0.89 \\
& verano & 0.20 & 0.27 & 0.75 \\
& otoño & 0.08 & 0.12 & 0.63 \\
& invierno & 0.09 & 0.07 & 1.22 \\
& primavera & 0.22 & 0.16 & 1.36 \\
\hline
\end{tabular}

En condiciones de verano a partir de ambas fuentes de observación se registraron los más altos valores de promedio y mediana, mientras que los más bajos se presentaron en ambas observaciones, correspondiendo al satélite en condiciones de invierno e in situ en otoño. Asociado a valores altos la mediana para condiciones de verano en base a observaciones in situ fue de $1.77 \mathrm{mg} \cdot \mathrm{m}^{-3}$ y vía satélite fue de 2.31 $\mathrm{mg} \cdot \mathrm{m}^{-3}$. Mientras que asociado a valores bajos la mediana vía satélite fue de $0.90 \mathrm{mg} \cdot \mathrm{m}^{-3}$ en invierno, y la mediana in situ fue de $0.96 \mathrm{mg} \cdot \mathrm{m}^{-3}$ en condiciones de otoño. (Figura 4).

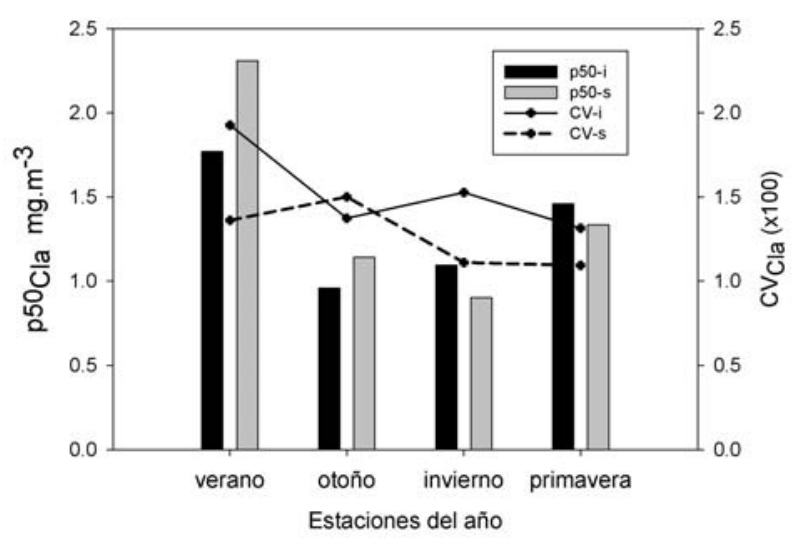

Figura 4. Comparación estacional de: Medianas (p50) de $\mathrm{Cla}_{\mathrm{i}}$ (barras negras) y $\mathrm{Cla}_{\mathrm{s}}$ (barras grises). Coeficientes de variabilidad (CV) de $\mathrm{Cla}_{\mathrm{i}}$ (línea sólida) y Cla $\mathrm{s}_{\mathrm{s}}$ (línea punteada), en pares coincidentes entre 1998-2007.

La comparación con el percentil de cada estación evidenció que cuando se hallan por debajo de p20 los valores satelitales son mayores que los observados in situ, en el p25 se presenta la misma característica a excepción de otoño. Valores de clorofila-a que se hallan alrededor de la mediana hasta el p75, evidencian que en condiciones de verano y otoño las observaciones vía satélite fueron mayores que las in situ mientras que en condiciones de invierno y primavera ocurrió lo contrario. Cuando se evaluó valores de clorofila-a por encima del p75, vía satélite se reportó menores concentraciones que las observaciones in situ, excepto en otoño.

La evaluación del coeficiente de variabilidad evidenció que, la variabilidad de $\mathrm{Cla}_{\mathrm{i}}$ fue más alta que la de $\mathrm{Cla}_{\mathrm{s}}$ en todas las estaciones del año, excepto en condiciones de otoño. (Figura 4), incluso en valores definidos como rango por el p1 a p99, p5 a p95 y p25 a p75.

Mientras que al evaluar los coeficientes de variabilidad en función a rangos definidos por la selección de observaciones in situ entre el siguiente percentil: p1 a p99, p2 a p98, hasta p5 a p95, las

Tabla 2. Parámetros estadísticos de asociación en datos coincidentes de clorofila-a evaluados vía satélite e in situ.

\begin{tabular}{|c|c|c|c|c|c|c|c|c|c|c|c|c|c|c|}
\hline Periodo & $\begin{array}{l}\text { Var: X \& Y } \\
\text { (sat \& in-situ) }\end{array}$ & $r(X, Y)$ & $r^{2}$ & $t$ & p & $\mathbf{N}$ & $\begin{array}{l}\text { Intercp } \\
\text { dep: } Y\end{array}$ & $\begin{array}{l}\text { Pend } \\
\text { dep: Y }\end{array}$ & $\begin{array}{l}\text { Intercp } \\
\text { dep: } X\end{array}$ & $\begin{array}{l}\text { Pend } \\
\text { dep: } X\end{array}$ & $\begin{array}{l}\text { RCME } \\
(\%)\end{array}$ & $\begin{array}{l}\mathrm{EM} \\
(\%)\end{array}$ & $\begin{array}{l}\log 10 \\
\text { RCME }\end{array}$ & $\begin{array}{c}\log 10 \\
E M\end{array}$ \\
\hline \multirow[t]{2}{*}{ General } & $\mathrm{Cla}$ & 0.49 & 0.24 & 12.86 & 0.00 & 527 & 1.2847 & 0.6811 & 1.6982 & 0.3517 & 168.12 & 44.40 & & \\
\hline & $\log 10$ (Cla) & 0.79 & 0.63 & 29.70 & 0.00 & 527 & -0.0033 & 0.9055 & 0.0726 & 0.6923 & & & 0.34 & 0.02 \\
\hline \multirow[t]{2}{*}{ verano } & $\mathrm{Cla}$ & 0.48 & 0.23 & 7.98 & 0.00 & 215 & 1.1514 & 0.7038 & 2.2896 & 0.3268 & 205.20 & 60.05 & & \\
\hline & $\log 10$ (Cla) & 0.82 & 0.67 & 20.73 & 0.00 & 215 & -0.0435 & 0.9083 & 0.1212 & 0.7361 & & & 0.33 & 0.07 \\
\hline \multirow[t]{2}{*}{ otoño } & $\mathrm{Cla}$ & 0.46 & 0.21 & 5.68 & 0.00 & 123 & 1.3422 & 0.3592 & 1.3500 & 0.5853 & 182.45 & 60.49 & & \\
\hline & $\log 10$ (Cla) & 0.72 & 0.52 & 11.55 & 0.00 & 123 & -0.0101 & 0.7154 & 0.0656 & 0.7332 & & & 0.37 & 0.04 \\
\hline \multirow[t]{2}{*}{ Invierno } & $\mathrm{Cla}$ & 0.84 & 0.71 & 10.20 & 0.00 & 44 & -0.4141 & 1.6538 & 0.7003 & 0.4308 & 113.96 & 30.36 & & \\
\hline & $\log 10$ (Cla) & 0.76 & 0.58 & 7.69 & 0.00 & 44 & 0.0124 & 1.0500 & 0.0241 & 0.5570 & & & 0.34 & -0.02 \\
\hline \multirow[t]{2}{*}{ Primavera } & $\mathrm{Cla}$ & 0.61 & 0.37 & 9.20 & 0.00 & 145 & 0.9620 & 1.1481 & 1.1320 & 0.3237 & 90.12 & 11.79 & & \\
\hline & $\log 10(\mathrm{Cla})$ & 0.84 & 0.71 & 18.80 & 0.00 & 145 & 0.0374 & 1.1272 & 0.0224 & 0.6315 & & & 0.31 & -0.06 \\
\hline
\end{tabular}


características que se describen de manera independiente cambian, observándose vía satélite mayor variabilidad que las observaciones in situ durante verano hasta otoño, y registrándose menor variabilidad durante invierno hasta primavera, frente a las observaciones in situ.

A diferencia de coeficientes de variabilidad evaluados en función a rangos definidos por la selección de observaciones vía satélite entre el siguiente percentil: p1 a p99, p2 a p98 y p3 a p97, las características de variabilidad descritas de manera independiente, se van acentuando cuanto más estrecho es el rango evidenciando desde las observaciones in situ mayor variabilidad en condiciones de verano y menor variabilidad en otoño, invierno y primavera. Estas características cambian cuando los rangos se definen entre los p5 a p95 estrechándose progresivamente hasta p25 a p75, observándose en valores in situ que la variabilidad aún es la más alta que las demás estaciones y que en invierno suele presentarse menor variabilidad, que en todas las demás estaciones. A diferencia de las observaciones vía satélite donde todos los casos de selección evidenciaron en otoño mayor variabilidad que en las demás estaciones del año, incluso llegando a registrar mayor variabilidad que las observaciones in situ cuando el rango basado en el percentil estuvo seleccionado entre p1 a p99 y p2 a p98.

\section{Discusión.}

El cociente de $\mathrm{Cla}_{\mathrm{i}}$ : $\mathrm{Cla}_{\mathrm{s}}$ promedio de valores transformados por $\log _{10}$ fue menor a 1 (Tabla 1 ), lo cual indica que las observaciones vía satélite sobreestiman la clorofila in situ, confirmando la primera hipótesis planteada, coincidiendo con investigaciones similares en otras regiones (Hyde et al, 2007). Estos resultados difieren del cociente $\mathrm{Cla}_{\mathrm{i}}$ : $\mathrm{Cla}_{\mathrm{s}}$ de 3.8 encontrado para el Perú por Chávez (1995) que indica que el satélite (CZCS) subestimó la clorofila in situ. Esta diferencia la atribuimos a la influencia de valores extremos del percentil de clorofila-a, dado que el cociente fue mayor a 1 con datos no transformados, pero fue menor a 1 al eliminar los datos que se hallan en los extremos del percentil, considerando los valores definidos en un rango más estrecho entre el p15 y el p85. La presencia de valores muy altos o muy bajos de clorofila-a están asociados a la pigmentación de fitoplancton que depende de la concentración intracelular de clorofila-a, respecto a su tamaño celular y a las condiciones de crecimiento (Staehr, 2002), por ende, a la composición de especies. Así como los pigmentos accesorios y feopigmentos (clorofila digerida), cuyos valores por lo general son muy altos en aguas costeras muy productivas (Ramírez \& Pizarro, 2005).

Otro de los factores que influyen en las diferencias de valores reportados vía satélite e in situ se debe al tipo de bandas espectrales registrados, CZCS fue destinado a medir el color del océano con 4 de las 6 bandas, de $20 \mathrm{~nm}$ de ancho, centradas en 443, 520, 550 y $670 \mathrm{~nm}$. Mientras que SeaWiFS, de las 8 bandas 4 con $20 \mathrm{~nm}$ de ancho centradas en los 443, 490, 510, $555 \mathrm{~nm}$ fueron destinadas para estimar clorofila-a, complementadas con bandas de $412 \mathrm{~nm}$ (para materia orgánica disuelta), $670 \mathrm{~nm}$ (para corrección atmosférica y sedimentos), 765 y 865 nm (para corrección atmosférica y radiación de aerosoles). Así como al tipo de algoritmo usado, tal es el caso que la comparación realizada al norte de Chile con el OC2 el cual solo utiliza dos bandas espectrales, determinaron que valores vía satélite (SeaWiFS), sobreestimaban en 2.36 veces más a la clorofila-a-in situ, cuyos resultados fueron similares a los obtenidos por Stramski \& Stramska (2001), quienes reportaron que SeaWiFS con el OC2 sobreestimó en 2.3 veces para los lagos de Noruega y Greenland (Silva et al., 2003).

Cabe mencionar que la primera versión del algoritmo OC4 (O'Reilly et al., 1998) fue formulada como una función polinomial cúbica modificada (MCP), sin embargo la actual versión del OC4 usa una función polinomial de cuarto orden. La ecuación MCP fue usada para refinar el OC2 con un mismo conjunto de valores $(\mathrm{N}=2804)$ usados para actualizar OC4. Con estas consideraciones reportaron que la comparación entre valores de clorofila-a modelados con OC2v4 y clorofila in situ tuvo un $\mathrm{r}^{2}$ de 0.883 con un RMS de 0.231. Mientras que comparaciones entre valores de clorofila-a modelados con OC4v4 y datos in situ, presentaron un $\mathrm{r}^{2}$ de 0.892 con un RMS de 0.222 . Frente a ello, sus resultados mostraron que el $\mathrm{r}^{2}$ obtenido con el algoritmo OC4 (0.892) fue ligeramente más alto que los obtenidos con el OC2 (0.883) (O’Reilly et al., 2000). Posteriores mejoras al algoritmo y métodos de procesamiento incorporados al cuarto reprocesamiento han mejorado notablemente la precisión de SeaWiFS en la estimación de clorofilaa (O'Reilly \& Yoder, 2003).

El error medio con $\log _{10}$, indicó que la $\mathrm{Cla}_{\mathrm{s}}$ sobreestimó a la $\mathrm{Cla}_{\mathrm{i}}\left(\mathrm{EM}_{\log }=0.02\right)$ (Tabla 2). Cabe señalar que las observaciones in situ reportan resultados de clorofila-a con una corrección por feopigmentos (Holm-Hansen et al., 1965), con valores más bajos que la clorofila-a total. Por otro lado, LeeBorges \& Armstrong (2002) mencionan que hay una pérdida de hasta $20 \%$ de clorofila a través de los filtros Whatman.

El cociente de medias con valores $\log _{10}$, en condiciones de verano y otoño, indicó que las observaciones vía satélite sobreestimaron la $\mathrm{Cla}_{\mathrm{i}}$ (Tabla 1), no obstante ello no concordó plenamente en la observación del cociente de medias sin transformación, hallándose en verano un valor mayor a 1. Sin embargo, al evaluar valores que estuvieron en un rango de percentil ligeramente más estrecho (p1 a p99), el cociente fue menor a 1 , ratificando los resultados obtenidos con una transformación $\log _{10}$. 
Los cocientes obtenidos para invierno y primavera indicaron que la $\mathrm{Cla}_{\mathrm{s}}$ subestimó la $\mathrm{Cla}_{\mathrm{i}}$.

Así mismo fueron congruentes con el error medio de clorofila-a transformada con $\log _{10}$ en verano $\mathrm{y}$ otoño $\left(\mathrm{EM}_{\log }=0.07\right.$ y 0.04 respectivamente), donde los valores mayores a cero indican que la $\mathrm{Cla}_{\mathrm{s}}$ sobreestimó la $\mathrm{Cla}_{\mathrm{i}}$, mientras que para invierno y primavera $\left(\mathrm{EM}_{\mathrm{log}}=-0.02\right.$ y -0.06 respectivamente) los valores vía satélite subestimaron los valores de $\mathrm{Cla}_{\mathrm{i}}$. Por otro lado hay evidencias que SeaWiFS sobreestima las bajas concentraciones de clorofila-a (< 0.2 mg. ${ }^{-3}$ ) (Lee-Borges \& Armstrong, 2002; Hyde et al, 2007), lo cual podría darse en zonas más oceánicas $\mathrm{y}$ en condiciones de invierno, pero los resultados muestran lo contrario.

Consideramos que las diferencias y su eficiencia, depende de las variaciones locales de las Propiedades Opticas Inherentes a los constituyentes del agua (IOCCG, 2000; Loisel et al, 2010). La presencia de destellos o reflejos solares en la superficie del océano (Bulgarelli et al, 2004) y aerosoles atmosféricos en zonas costeras merece especial atención, y exploración para el desarrollo de nuevos algoritmos a nivel regional. Los aerosoles atmosféricos son suspensiones de partículas sólidas o líquidas en el aire, siempre presentes en el aire y a menudo son observables como polvo, humo y neblina (Kahn et al, 2009).

El coeficiente de determinación hallado a nivel general fue alto entre los valores transformados de $\mathrm{Cla}_{\mathrm{i}}$ y Cla $\mathrm{Cl}_{\mathrm{s}}\left(\mathrm{r}^{2}=0.63\right)$ en comparación a otros estudios, como Rodríguez-Guzmán \& Gilbes-Santaella (2009), que al comparar sólidos suspendidos totales in situ y vía satélite (MODIS) encontraron un coeficiente de determinación menor $\left(\mathrm{r}^{2}=0.40\right)$. Por otro lado se sabe que los datos del Coastal Zone Color Scanner (CZCS) no proveyeron una representación precisa de la concentración de clorofila-a en el Perú por errores de corrección atmosférica (Chávez, 1995), dado que se encontró que con el CZCS la precisión de la biomasa fue más o menos $35 \%$, por otro lado la biomasa en el océano varía por cuatro órdenes de magnitud, los resultados de la información obtenida a gran escala deben considerarse apreciables (Sathyendranath \& Platt, 1993).

La mejor bondad de ajuste estuvo definida por el valor más bajo de la RCME, correspondiendo a condiciones de primavera $\left(\mathrm{RCME}_{\mathrm{log}}=0.31\right)$, seguido por las condiciones de verano $\left(\mathrm{RCME}_{\log }=0.33\right)$ (Tabla 2). Por lo tanto, la segunda hipótesis planteada no fue confirmada. Los errores altos antes de la transformación logarítmica fueron coincidentes con resultados de investigaciones similares en otras regiones. El RCME $E_{\log }=0.34$ hallado en condiciones de invierno fue bastante cercano al encontrado en los meses de Julio a Agosto (0.35) en otras regiones como China (Zhang et al., 2006). Considerando la menor nubosidad en verano, la mejor bondad de ajuste en primavera la atribuimos a la mayor variabilidad de la clorofila in situ en condiciones de verano, mucho más alta que en las demás estaciones del año, posiblemente debido a la mayor frecuencia de floraciones algales en verano, sin dejar de lado la resolución espacial de los datos obtenidos vía satélite (SeaWiFS), con los cuales evidentemente no se llega a captar la variabilidad espacial que se registra en las mediciones in situ, dado que la alta variabilidad de propiedades bio-òpticas junto a diferencias en técnicas de medición (pixel versus punto) contribuyen a inconsistencias entre propiedades bio-ópticas percibidas in situ $\mathrm{y}$ remotamente (Chang \& Gould, 2006).

\section{Conclusiones.}

En general, los datos obtenidos vía satélite sobreestiman ligeramente la clorofila in situ, como indica el cociente de medias transformadas logarítmicamente $\left(\mathrm{Cla}_{\mathrm{i}}: \mathrm{Cla}_{\mathrm{s}}=0.89\right)$ y el error medio $\left(\mathrm{EM}_{\log }=0.02\right)$, lo cual confirmó la primera hipótesis planteada.

Por otro lado, vía satélite en condiciones de verano se sobreestimó la concentración de clorofila-a in situ, y en condiciones de invierno la subestimó.

La segunda hipótesis que plantea en condiciones de verano un mejor ajuste entre la $\mathrm{Cla}_{\mathrm{s}}$ y la $\mathrm{Cla}_{\mathrm{i}}$, no fue confirmada, ya que en primavera se alcanzó el mayor coeficiente de correlación $(r=0.84)$.

La ecuación general que permite estimar valores de $\mathrm{Cla}_{\mathrm{i}}$ a partir de $\mathrm{Cla}_{\mathrm{s}}$ frente a Perú es la siguiente:

$$
\mathrm{Cla}_{\mathrm{i}}=0.9925 \mathrm{Cla}_{\mathrm{s}}{ }^{0.9055}
$$

\section{Agradecimientos.}

Agradecemos al Instituto del Mar del Perú, por haber brindado las condiciones, para el levantamiento de la información in situ, la obtención de información satelital y la elaboración de ésta investigación.

\section{Literatura citada.}

Bulgarelli B., Mélin F. \& Zibordi G. 2004. Atmospheric correction of SeaWiFS data. In: Wolfram S (ed). Validation of algorithms for chlorophyll a retrieval from satellite data in the baltic sea area. Baltic Sea Environment Proceedings No94: 11-22.

Calienes R., Guillen O. \& Lostaunau N. 1985. Variabilidad espacio-temporal de clorofila, producción primaria y nutrientes frene a la costa peruana. Bol. Inst. Mar / OEA. Vol. 10(9).

Chang G.C. \& Gould R.W. 2006. Comparisons of optical properties of the coastal ocean derived from satellite ocean color an in situ measurements. Optics express 14 (22): 10149-10163.

Chin M., Kahn R.A. \& Schwartz S.E. (eds.). 2009. Atmospheric Aerosol Properties and Climate Impacts, A Report by the U.S. Climate Change Science Program and the Subcommittee on Global Change Research. National Aeronautics and Space Administration, Washington, D.C. USA, 128 pp. 
Chávez F. 1995. A comparison of ship and satellite chlorophyll from California and Peru, J. Geophys. Res., 100: 24,855-24,862.

Echevin V., Aumont O., Ledesma J. \& Flores G. 2008. The seasonal cycle of surface chlorophyll in the Peruvian upwelling system: A modelling study. In: Werner F., \& Lough R.G. (eds). The Northern Humboldt Current System: Ocean Dynamics, Ecosystem Processes, and Fisheries. Progress in Oceanography 79: 167-176.

Flores G., Córdova J., Ledesma J. \& Robles C. 1998. Características químicas y de clorofila "a" del mar peruano durante el otoño 1998. Crucero BIC Humboldt 9803-05 de Tumbes a Tacna. Inf Inst. Mar Perú 135: 6778.

Flores G., Carrasco N. \& Sarmiento M. 2001. Oxígeno disuelto, nutrientes y clorofila-a del mar peruano en mayo 2000. Inf Inst. Mar Perú. 163.

Holm-Hansen O., Lorenzen C.J., Holms R.W. \& Strickland J.D.H. 1965. Fluorometric Determination of Chlorophyll. J. Cons.perm.int Explor. Mer. 30: 3-15.

Hyde K.J.W., O'Reilly J.E. \& Oviatt C.A. 2007. Validation of SeaWiFS chlorophyll a in Massachusetts Bay. Continental Shelf Research 24: 1677-1691.

IOCCG. 2000. Remote sensing of Ocean Colour in Coastal, and Other Optically-Complex, Waters. Sathyendranath S. (ed.) Report of the Interantional Ocean-Colour Coordinating Group, No 3, IOCCG, Darmounth, Canada.

Kahn RA., Yu H., Schwartz S.E., Chin M., Feingold G., Remer L.A., Rind D., Halthore R. \& DeCola P. 2009. Introduction, in Atmospheric Aerosol Properties and Climate Impacts, A Report by the U.S. Climate Change Science Program and the Subcommittee on Global Change Research. In: Chin M, Kahn RA, Schwartz SE, (eds.). National Aeronautics and Space Administration, Washington, D.C., USA.

Lee-Borges J. \& Armstrong R. 2002. SeaWiFS Validation at the Caribbean time series station (cats). On line: $<$ http://gers.uprm.edu/pdfs/leeborges_etal_02.pdf >

Loisel H., Lubac B., Dessailly D., Duforet-Gaurier L. \& Vantrepotte V. 2010. Effec of inherent optical properties variability on the chlorophyll retrieval from ocean color remote sensing: an in situ approach. Optics Express Vol.18(20): 20949-20959

Maritorena S., Siegel D.A. \& Peterson A.R. 2002. Optimization of a semianalytical ocean color model for globalscale applications. Appl. Opt. 41(15), 2705-2714.

Marrari M., Hu C. \& Daly K. 2006. Validation of SeaWiFS chlorophyll a concentrations in the Southern Ocean: A revisit. Remote Sensing of Environment 105: 367-375.

O’Reilly J.E., Maritorena S., Siegel D., O’brien M.C., Toole D., Mitchell B.G., Kahru M., Chavez F.P., Strutton P., Cota G., Hooker S.B., McClain C.R., Carder K.L., Muller-Karger F., Harding L., Magnuson A., Phinney D., Moore G.F., Aiken J., Arrigo K.R., Letelier R. \& Culver M. 2000. Ocean color chlorophyll a algorithms for SeaWiFS, OC2, and OC4: Version 4. In: Hooker S. B. and E. R. Firestone (Eds.), SeaWiFS Postlaunch Technical Report Series, SeaWiFS Postlaunch Calibration and Validation Analyses, Volume 11, Part 3, 9-23, Greenbelt, Maryland: NASA Goddard Space Flight Center, 1-51.

O’Reilly J.E. \& Yoder J.A. 2003. A comparison of SeaWiFS LAC products from the third and fourth reprocessing:
Northeast US ecosystem. In: Patt., F.S., Barnes, R.A., Eplee Jr., R.E., Franz B.A., Robinson W.D., Feldman G.C., Bailey S.W., Gales J., Werdell P.J., Wang M., Frouin R., Stumpf R.P., Arnone R.A., Gould Jr. R.W., Martinolich P.M., Ransibrahmanakul V., O’Reilly J.E. \& Yoder J.A. (Eds.), Algorithm Updates for the Fourth SeaWiFS Data Reprocessing. NASA Goddard Space Flight Center, Greenbelt, MD, pp. 60-67.

Pan Y., Tang D. \& Weng D. 2010. Evaluation of the SeaWiFS and MODIS Chlorophyll a algorithms used for the Northern South china Sea during the Summer Season. Terr. Atmos. Ocean. Sci., Vol. 21, Nº 6, 9971005.

Pizarro O., Hormazábal S., Gonzáles A. \& Yañez E. 1994. Variabilidad del viento, nivel del mar y temperatura en la costa norte de Chile. Invest. Mar., Valparaíso, 22:85101.

Pennington J., Mahoney K., Kuwahara V., Kolber D., Calienes R. \& Chavez F. 2006. Primary production in the eastern tropical Pacific: A review. Progress in Oceanography 69: 285-317.

Ramírez B. \& Pizarro E. 2005. Distribución de clorofila-a y feopigmentos en los canales australes chilenos comprendidos entre Puerto Montt y la laguna San Rafael, Chile. Ciencia y Tecnología del Mar Vol.28(001): 45-62.

Rodríguez-Guzmán V. \& Gilbes-Santaella F. 2009. Using MODIS $250 \mathrm{~m}$ imagery to estimate total suspended sediment in a tropical open bay. International Journal of System Applications, Engineering \& Development 1(3), 2009 on line: $<$ http://gers.uprm.edu/pdfs/rodriguez_gilbes_09b.pdf >

Staehr P.A., Markager S. \& Sand_Jensen K. 2002. Optical properties of marine phytoplankton. Thesis $\mathrm{PhD}$. University of Copenhagen.

Stramski D \& Stramska M. 2001. Optical and ancillary measurements at high latitudes in support of the MODIS ocean validation program. NASA Grant Nag5-6466, Final Report.

Sathyendranath S. \& Platt T. 1993. Remote sensing of water-column primary production. In: $\mathrm{Li}$ WKW, Maestrini SY, (eds). Measurement of Primary Production from the Molecular to the Global Scale. Vol. 197. Copenhagen: ICES Marine Science Symposia, p 236-243.

Silva C., Yañez E., Barbieri M. \& Nieto K. 2003. Asociación entre la pesquería de pequeños pelágicos, la clorofila a y la temperatura superficial del mar en la zona norte de Chile. (ed) Yañez E. Actividad pesquera y de acuicultura en Chile Ediciones Universitarias de Valparaíso: 157-162.

Sin-Jae Y. 2002. Validation of chlorophyll algorithm un Ulleung Basin, East/Japan Sea. Korean Journal of Remote Sensing, Vol. 18(1): 35-42. $<$ http://ieg.or.kr/abstract/E180104.PDF>

Strickland J.D.H. \& Parsons T.R. 1968. A practical handbook of seawater analysis. Pigment analysis, Bull. Fish. Res. Bd. Canada, 167.

Thomas A.C., Carr M-E. \& Strub PT. 2001. Chlorophyll variability in eastern boundary currents. Geophysical Research Letters, Vol. 28(18): 3421-3424.

Werdell P.J., Bailey S.W., Franz B.A., Morel A. \& McClain C.R. 2007. On-orbit vicarious calibration of ocean color 
D. QUISPE, R. CALIENES, J. TAM, M. GRACO, J. LEDESMA, G. FLORES, D. CORREA Y C. QUISPE

Ecol. apl. Vol. $9 \mathrm{~N}^{\mathrm{O}}$ 2, pp. 151-159

sensors using an ocean surface reflectance model. Appl. Opt. 46(23), 5649-5666.

Zhang C., Hu C., Shang S., Muller-Karger F.E., Li Y., Dai M., Huang B., Ning X. \& Hong H. 2006. Bridging between SeaWiFS and MODIS for continuity of chlorophyll-a concentration assessments off Southeastern China. Remote Sensing of Environment 102: 250-263.

\footnotetext{
${ }^{1}$ Centro de Investigaciones en Modelado Oceanográfico y Biológico Pesquero, Instituto del Mar del Perú, P.O. Box 22, Callao, Perú. E-mail: dquispe@imarpe.pe

${ }^{2}$ Unidad de Hidroquímica, Instituto del Mar del Perú, P.O. Box 22, Callao, Perú.
} 